\title{
ÉTUDE COMPARÉE DE LA VAGUE SPERMATOGÉNÉTIQUE CHEZ LE TAUREAU ET CHEZ LE RAT
}

\author{
Marie-Thérèse IIOCHEREAU \\ Station Centrale de Physiologie animale, \\ Centre national de Recherches zootechniques, Jouy-en-Josas (Seine-et-Oise)
}

\section{SOMMAIRE}

Nous avons étudié chez le Taureau et chez le Rat la succession des stades du cycle de l'épithélium séminifère le long des tubes séminifères par la technique de reconstitution de ces tubes sémi. nifères sur coupes histologiques sériées. Nos résultats ont mis en évidence une continuité dans la succession numérique des stades et l'existence de la vague spermatogénétique définie par REGAUD. Cependant celle-ci peut présenter des modulations qui sont des inversions locales de l'ordre de succession des stades sans discontinuité dans l'ordre numérique de succession de ces stades.

La longueur de segments de tubes occupée par un stade déterminé en différents endroits d'un même tube ou sur des tubes différents est variable. Il n'y a donc pas de relation entre les longueurs individuetles des différents segments des tubes séminiféres occupées par un stade déterminé et la durée de ce stade qui est constante. Mais il existe une correspondance entre la longueur moyenne relative de chaque stade et sa fréquence relative calculée par comptage sur coupes histologiques.

\section{INTRODUCTION}

La plupart des auteurs admettent que chez les Mammifères les différents processus spermatogénétiques, depuis la multiplication des spermatogonies souches jusqu'à l'élimination des spermatozoïdes dans la lumière du tube séminifère, se succèdent et se répètent dans le temps de façon régulière.

Ceci s'explique par le fait que, d'une part, la " néoformation de nouvelles lignées spermatiques se poursuit sur toute la surface interne de la membrane du tube, sans attendre que les lignées précédentes aient achevé leur évolution et aient disparu sous forme de spermatozoïdes " (REGAUD, IgOI); mais surtout la néoformation d'une lignée est reliée de façon précise à l'évolution des autres générations cellulaires situées dans la même section orthogonale du tube séminifère.

De cette évolution régulière et coordonnée des différentes générations cellulaires de la lignée spermatogénétique découle la formation d'associations cellulaires ou stades dont la répétition forme le cycle de l'épithélium séminifère. On peut penser 
que de même le long du tube les différents stades se succèdent dans le même ordre que dans le cycle de l'épithélium séminifère. Cette hypothèse est à la base de la notion de vague spermatogénétique proposée par VON EBNER (I87I) et définie par REGAUD (IgOI) de la façon suivante "la vague spermatogénétique est dans l'espace ce que le cycle de l'épithélium séminifère est dans le temps ».

L'existence de cette vague a été grossièrement vérifiée par VON EBNER (I87I et I888) chez le Rat, par BENDA (I887) chez différents Mammifères (Lapin, Rat, Taureau, Bélier), par Furst (I887) chez les Marsupiaux et par CurTrs (I9I8) chez le Lapin, le Chien, et la Souris.

REGAUD (IgOI) a même pensé que les différents stades du cycle de l'épithélium séminifère occupaient dans le tube une longueur proportionnelle à leur durée ; la longueur de la vague spermatogénétique complète devait donc être proportionnelle à la durée du cycle de l'épithélium séminifère.

Cependant cette conception rigide de la vague spermatogénétique a été contestée par de nombreux auteurs.

Déjà Von EBNER (I87I) avait observé chez le Rat la présence de vagues spermatogénétiques de longueurs variables. En outre de nombreuses irrégularités dans l'ordre de succession des stades ont été rapportées chez le Lapin, le Chien et la Souris par CurTis (I9I8) et chez le Taureau par Kramer (I960). Mais si les auteurs précédents admettent l'existence d'une vague spermatogénétique même perturbée, CLELAND (I95I) nie complètement l'existence de celle-ci chez le Cobaye. Cet auteur conclut à l'absence de " séquence régulière des stades le long du tube séminifère " le tube est divisé en unités qui évoluent indépendamment les unes des autres empêchant toute organisation d'ensemble de la spermatogenèse et l'existence de vagues spermatogénétiques le long du tube séminifère.

Il est vraisemblable qu'une telle divergence d'opinions peut résulter de l'utilisation soit de techniques de reconstruction des tubes séminifères trop grossières soit d'une définition insuffisante des associations cellulaires.

En effet, les résultats obtenus récemment par PEREY, CLERMONT et LEBLOND (I96I) chez le Rat en utilisant plusieurs techniques de reconstitution des tubes séminifères et une classification précise des stades du cycle de l'épithélium séminifère, ont montré que la vague spermatogénétique existait réellement bien que la présence de "modulations » ait pu par endroit faire douter de sa réalité.

Dans le cadre d'une étude générale de la spermatogenèse chez les animaux domestiques, nous avons effectué une étude de la vague spermatogénétique chez le Taureau en comparant nos résultats avec ceux obtenus chez le Rat.

\section{MATÉRIEI ET MÉTHODE}

\section{$\left.\mathrm{I}^{\circ}\right)$ Animaux étudiés}

Nos études ont été effectuées sur 4 taureaux de 2 à 6 ans, donc pubères et appartenant aux races françaises Pie rouge de l'Est, Normande et Charolaise. L'examen préalable des éjaculats récoltés au vagin artificiel avait montré la présence de sperme en quantité et qualité normales.

Les 4 rats, âgés de 3 mois, étaient des Wistar de souche " 03 Jouy-en-Josas "; la fertilité de ces animaux avait été contrôlée par accouplement. 


\section{$\left.2^{\circ}\right)$ Techniques histologiques}

Les testicules des taureaux ont été prélevés dans les cinq minutes qui suivent l'abattage en conservant une partie du cordon testiculaire pour effectuer une préfixation par perfusion, rendue nécessaire par le besoin de prélever de gros blocs allant du rete-testis à l'albuginée. Une première perfusion de sérum physiologique à $37^{\circ} \mathrm{C}$ dans l'artère testiculaire est suivie immédiatement après élimination du sang par une deuxième perfusion à l'aide du liquide de fixation : 100 à $200 \mathrm{ml}$ sont nécessaires dans ce cas. Les testicules de rats furent prélevés et fixés dans leur totalité.

Après essai de différents fixateurs, nous avons finalement retenu un fixateur de composition proche de celle du HELLY mais dont la concentration en bichromate de $K\left({ }^{1}\right)$ a été diminuée pour réduire la contraction des tubes séminifères. La fixation à la température ambiante dure 26 heures pour les testicules de Rat et 36 heures pour les fragments de testicule de Taureau.

$\mathrm{La}$ déshydratation à l'alcool éthylique doit être très progressive et se termine par des bains de benzoate de méthyle, avant une inclusion classique. La durée de cette inclusion varie de 33 heures pour le Rat à 42 heures environ pour le Taureau.

Les coupes sériées de $15 \mu$ d'épaisseur pour le Taureau et de Io $\mu$ d'épaisseur pour le Rat ont été colorées au réactif de Schiff, suivant la coloration de Feulgen et au bleu Alcyan (Mowry, I960).

\section{$\left.3^{\circ}\right)$ Reconstitution des tubes séminifères}

Nous avons procédé à la reconstitution de tubes séminifères in situ en déterminant la nature et la longueur des différents stades du cycle de l'épithélium séminifère, soit directement sur coupes histologiques, soit sur photographies de celles-ci.

a) Le type de classification utilisé a été celui défini par CURTIS (I918), Roosen RUNGE et GiESEL (I950), ORTAVANT (1958) et AMMAN (I961) car il permet de suivre facilement les grands événements de la spermatogenèse : division méiotique, transformation des spermatides, libération des spermatozoïdes; nous avons défini 8 stades (planches nos I à ro).

b) La longueur d'un segment quelconque de tube séminifère a été confondue avec celle de son axe longitudinal.

Les sections des tubes séminifères repérées dans la coupe histologique sont circulaires ou elliptiques selon que le plan de coupe est orthogonal ou oblique par rapport à l'axe longitudinal du tube. Elles proviennent soit de portions grossièrement rectilignes, soit de portions situées au niveau de circonvolutions. Nous avons cherché à déterminer la longueur de l'axe longitudinal du tube dans chaque cas.

I. - Dans le cas de portion rectiligne de tube séminifère :

- si la section est circulaire, la longueur de l'axe du tube se confond en fait avec l'épaisseur de la coupe ;

- si la section est elliptique, cette longueur doit être calculée en fonction de l'obliquité du plan de coupe. A cet effet on peut représenter le segment de tube séminifère dans le plan passant par l'axe du tube et le grand axe de l'ellipse selon le schéma de la figure $\mathbf{I}$.

Les règles classiques de la géométrie des triangles semblables nous ont permis de déduire la formule suivante :

$$
\mathrm{L}=\frac{\mathrm{D} \times e}{d}
$$

dans laquelle: $\quad \mathrm{L}=$ longueur du fragment de tube étudié ;

$\mathrm{D}=$ grand axe de la section elliptique;

$d=$ petit axe de la section elliptique;

$e=$ épaisseur de la coupe.

2. - Dans le cas de portion de tube située au niveau d'une circonvolution, nous avons assimilé chaque branche $\mathrm{A}$ et $\mathrm{B}$ du coude à un tube rectiligne coupé obliquement et représenté dans le plan passant par l'axe du tube et le grand axe de l'ellipse, par un parallélogramme MKPI (fig. 2). La longueur de l'axe $\mathrm{DC}=1$ de la branche du coude $\mathrm{A}$, peut être confondue approximativement avec celle du segment $\mathrm{DI}$ égale à la moitié de la médiane $\mathrm{DD}^{\prime}=1$ du parallélogramme $\mathrm{MKPH}$; cependant l'erreur commise est faible. La hauteur PJ de ce parallélogramme est égale au produit de l'épaisseur de chaque coupe par le nombre $n$ de coupes situées entre le point de jonction $\mathrm{K}$ des deux branches $\mathrm{A}$ et $\mathrm{B}$ du tube et le point de disparition $\mathrm{L}$ de la membrane basale du tube.

(1) 1 ooo cc eau distillée; $50 \mathrm{~g}$ sublimé mercurique; $10 \mathrm{~g}$ bichromate de $\mathrm{K}$; $10 \mathrm{~g}$ de sulfate de Na. Au moment de l'emploi $3 \circ \mathrm{p}$. 100 de formol neutre, $5 \mathrm{p}$. 100 acide acétique. 


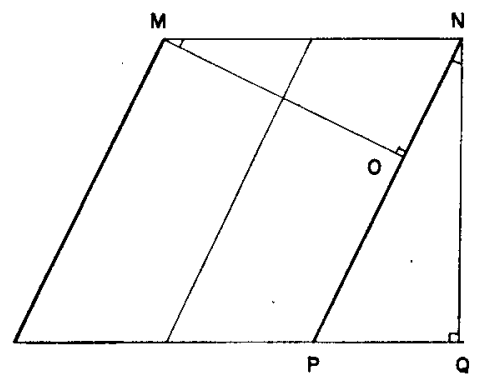

$$
\begin{aligned}
\text { FIG. I. }-\mathrm{MN}=\text { grand axe de l'ellipse }=\mathbf{D} ; \\
\mathrm{MO}=\text { diamètre réel du tube }=\text { petit axe de l'ellipse }=d \\
\mathrm{NP}=\text { longueur de fragment de tube étudié }=\mathbf{L} ; \\
\mathrm{NQ}=\text { épaisseur de la coupe }=e .
\end{aligned}
$$

Les règles des triangles semblables nous amènent à définir de même la formule suivante :

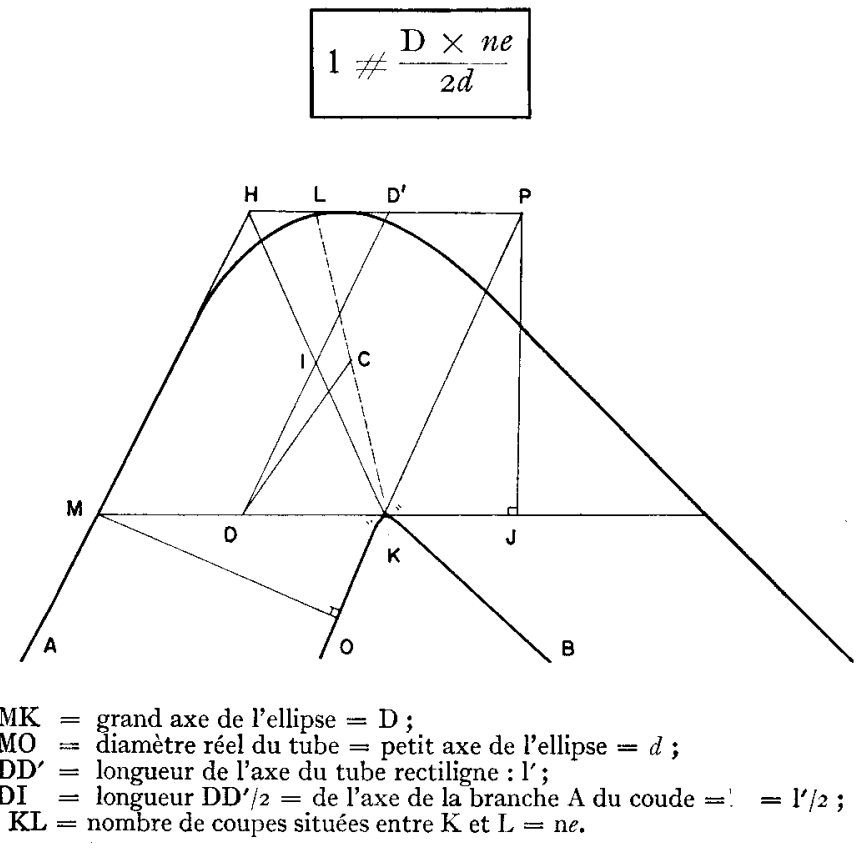

dans laquelle : $\mathbf{D}=$ grand axe de la section elliptique de la branche $\mathrm{A}$;

$d=$ petit axe de la section elliptique ;

$e=$ épaisseur de la coupe.

c) La longueur du segment de tube séminifère occupé par un stade donné est calculée d'après le nombre de sections sériées du tube sur lesquelles ce stade est représenté, en tenant compte des facteurs de correction établis précédemment. Lorsque deux stades successifs de la classification sont représentés sur la même section, nous avons compté la moitié de la longueur du segment de tube occupé conjointement par les deux stades présents, dans le calcul de la longueur de chacun de ces deux stades.

d) Les fréquences relatives des stades du cycle de l'épithélium séminifère ont été déterminées en comptant les sections orthogonales des tubes séminifères. 


\section{RÉSULTATS}

Iํ) RÉPARTITION DES STADES DU CYCLE DE L'ÉPITHÉLIUM SÉMINIFÈRE LE LONG DES TUBES

Chez le Rat, les tubes séminifères s'ouvrent dans le rete testis par les deux extrémités; leur diamètre est d'environ $250 \mu$. Ils présentent de nombreuses circonvolutions séparées par des portions rectilignes longues de 2 à $5 \mathrm{~mm}$; généralement les tubes ne présentent pas de ramification, cependant nous avons trouvé dans un cas l'existence d'une branche latérale (fig. 3). Ce phénomène a été également signalé par Cl,ermon't et Huckins (I96I).

Chez le Taureau, les tubes séminifères diffèrent principalement de ceux du Rat par une multiplication des circonvolutions. Les portions rectilignes sont beaucoup plus courtes, en général de 0,9 à $\mathrm{I}, 2 \mathrm{~mm}$. Le diamètre moyen des tubes est de $230 \mu$ environ.

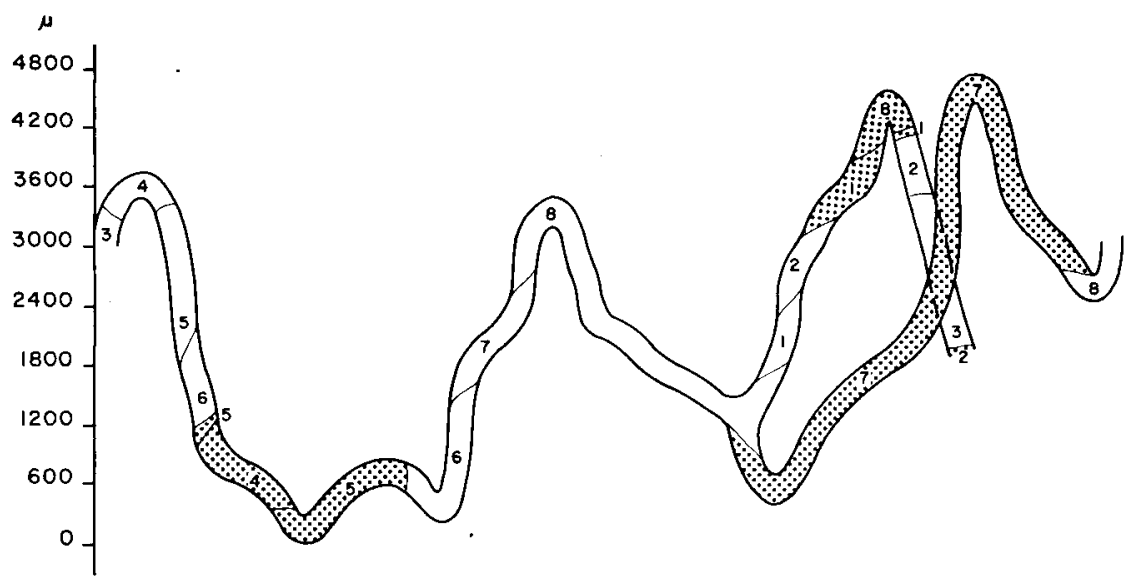

FIG. 3. - Segment de tube séminitère de Rat possédant un embranchement; malgré l'existence de cet embranchement on n'observe pas de discontinuité dans l'ordre de succession des stades

\section{a) Existence de la vague spermatogénétique}

Une vague spermatogénétique est caractérisée par la succession le long du tube dans un ordre croissant ou décroissant d'une série complète des différents stades du cycle de l'épithélium séminifère (définis précédemment) avec retour au stade de départ ; par exemple, si nous partons d'un stade $x$ nous devons rencontrer par définition dans l'ordre les stades $2,3,4,5,6,7,8$ et I ou les stades $8,7,6,5,4,3,2$, I. Ceci est valable quel que soit le stade de départ. Effectivement chez le Taureau et chez le Rat, nous avons constaté l'existence de telles vagues le long des tubes séminifères que nous avons reconstitués (fig. 4). Jamais nous n'avons observé de discontinuité dans l'ordre de succession des stades; chaque stade précède et succède un stade portant un numéro immédiatement voisin dans la classification. 


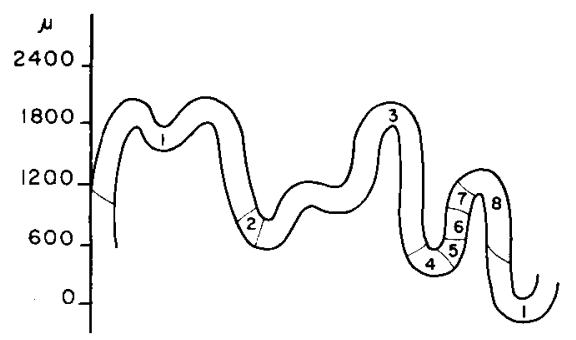

FIG. 4. - Segment de tube séminifère de Taureau présentant une vague spermatogénétique complète sans aucune perturbation. L'ordre de succession des stades est croissant de la gauche vers la droite

\section{b) Existence d'irrégularités à l'intérieur de la vague spermatogénétique}

Cependant, localement, l'ordre de succession des stades peut être perturbé par des irrégularités : en effet, sur une courte longueur de tube séminifère, cet ordre peut être localement inversé sans que pour cela que les numéros de stades ne soient pas immédiatement voisins puis il reprend son cours normal. On définit par modulation (PEREY, CleRmont et LEBLOND, I $96 \mathbf{I}$ ), une inversion de l'ordre de succession des stades intercalés dans une série croissante ou décroissante : par exemple dans la figure 5 , nous voyons une succession des stades décroissant de la gauche vers la droite sauf dans les deux zones ombrées où cet ordre est localement croissant.

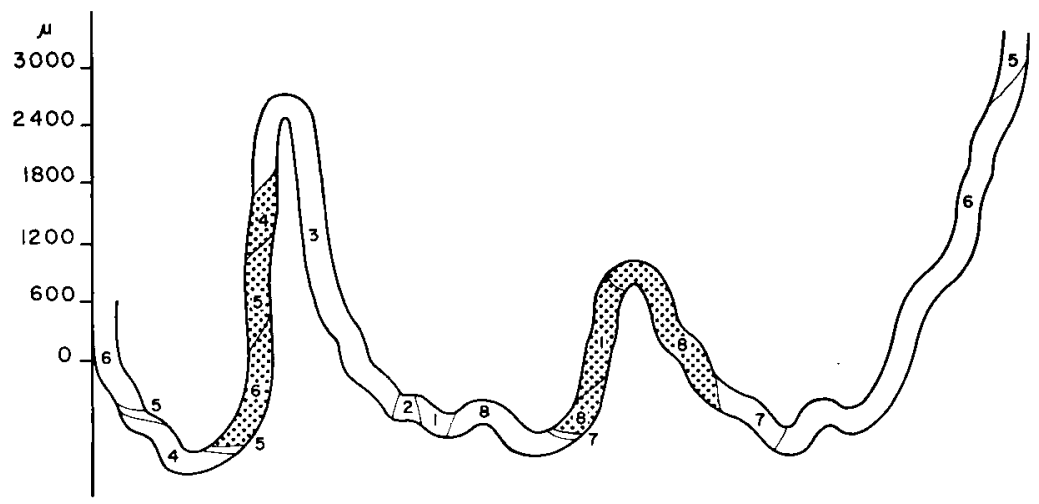

FIG. 5. - Segment de tube séminifère de Rat montrant 2 modulations (en gris sur la figure) portant chacune sur 3 stades. L'ordre de succession des stades est décroissant de la gauche vers la droite sauf au niveau des modulations, la longueur des segments occupés par un même stade est très variable, stade 6.

L'importance de cette modulation est variable : elle peut porter soit sur un seul stade (fig. 8), soit sur plusieurs stades (fig. 5) et se rencontre dans les deux espèces. Il résulte de la continuité dans l'ordre de succession des stades que si la modulation met en cause plusieurs de ceux-ci, leur ordre de succession à l'intérieur de celle-ci s'inverse toujours pour retrouver le numéro du stade précédant immédiatement le début de la modulation. Ainsi une modulation porte obligatoirement sur un nombre impair de stades. Nous en avons trouvé en effet portant sur I ou 3 stades chez le Rat et allant jusqu'à 7 stades chez le Taureau. Nos observations correspondent à ce que PEREY, LEBLOND et CleRMONT (I96I) ont observé chez le Rat. 


\section{$2^{\circ}$ ) LONGUEUR DES SEgMENTS DE TUBE OCCUPÉS PAR CHACUN DES STADES DU CYCLE DE L,'ÉPITHÉLIUM SÉMINIFÈ,RE}

Après avoir examiné la répartition spatiale des stades du cycle de l'épithélium séminifère le long des tubes, nous avons déterminé la longueur des segments de tubes occupés par chaque stade. Les résultats sont consignés dans les tableaux I et 2.

Les longueurs individuelles des différents segments occupés par un stade déterminé sont très variables à l'intérieur d'un testicule, non seulement d'un tube à l'autre mais également le long d'un même tube séminifère. Ceci est valable pour les deux

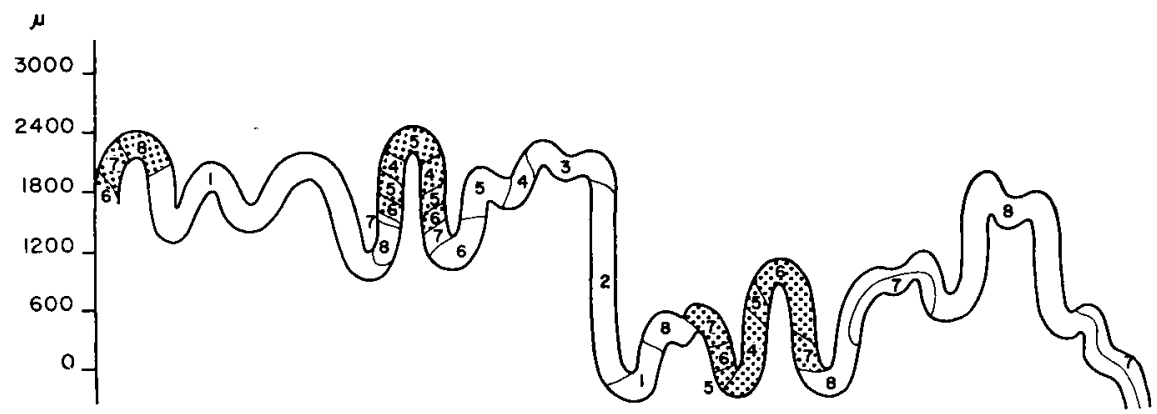

Fig. 6. - Segment de tube séminifère de Taureau. L'ordre de succession des stades est décroissant de la gauche vers la droite atec des modulations importantes (en gris sur la figure) ; la comparaison des figures 5 et 6 monire la différence de morphologie des tubes séminifères entre les 2 espèces.

espèces étudiées (fig. 3 et 5 pour le Rat, 6 et 7 pour le Taureau). Ainsi chez le Taureau la longueur du stade 4 peut varier de $60 \mu$ à I $720 \mu$, soit une variation de I à 28 et chez le Rat de $540 \mu$ à I $580 \mu$ soit de I à 3 .

\section{TABLEAU I}

Comparaison des longueurs relatives des stades du cycle de l'épithélium séminifère et des fréquences relatives de ces stades déterminées directement sur coupes histologiques chez le Taureau

\begin{tabular}{|c|c|c|c|c|c|c|c|}
\hline Stades & $\begin{array}{l}\text { plus petite } \\
\text { longueur }(\mu) \\
\text { de tube oc- } \\
\text { cupé par un } \\
\text { stade }\end{array}$ & $\begin{array}{l}\text { plus grande } \\
\text { longueur }(\mu) \\
\text { de tube oc- } \\
\text { cupé par un } \\
\text { stade }\end{array}$ & $\begin{array}{l}\text { longueur } \\
\text { moyenne }(\mu) \\
\pm \text { erreur type }\end{array}$ & $\begin{array}{l}\text { nombre de } \\
\text { données }\end{array}$ & $\begin{array}{l}\text { Coefficient } \\
\text { de variation }\end{array}$ & $\begin{array}{c}\text { proportion } \\
\text { relative } \% \\
\text { longueurs } \\
\text { moyennes }\end{array}$ & $\begin{array}{c}\text { fréquence } \\
\text { relative en } \\
\% \text { (par la } \\
\text { méthode } \\
\text { directe) } \\
\pm \text { erreur type } \\
72^{\prime} \text { sections }\end{array}$ \\
\hline $1 \ldots \ldots$ & 157 & 2937 & $1394 \pm 470$ & 6 & 33,7 & 32,1 & $27,59 \pm 0,66$ \\
\hline $2 \ldots \ldots$ & 187 & 320 & $288 \pm 33$ & 4 & 23,2 & 6,6 & $9,15 \pm 0,32$ \\
\hline $\mathbf{3} \ldots \ldots$ & 220 & 1620 & $703 \pm 92$ & 15 & 49,3 & 16,2 & $19,01 \pm 0,99$ \\
\hline $4 \ldots \ldots$ & 60 & 1721 & $539 \pm 108$ & 19 & 87 & 12,4 & $11,23 \div 0,88$ \\
\hline $5 \ldots \ldots$ & 30 & 454 & $145 \pm 26$ & 20 & 82,7 & 3,3 & $3,96 \pm 0,30$ \\
\hline $6 \ldots \ldots$ & 50 & 726 & $272 \pm 51$ & 13 & 63,9 & 6,2 & $9,39 \pm 0,53$ \\
\hline $7 \ldots \ldots$ & 90 & 853 & $359 \pm 82$ & 11 & 72,0 & 8,2 & $8,78 \pm 0,10$ \\
\hline $8 \ldots \ldots$ & 198 & 1135 & $638 \pm 91$ & 10 & 63,8 & 14,7 & $10,91 \pm 0,37$ \\
\hline
\end{tabular}




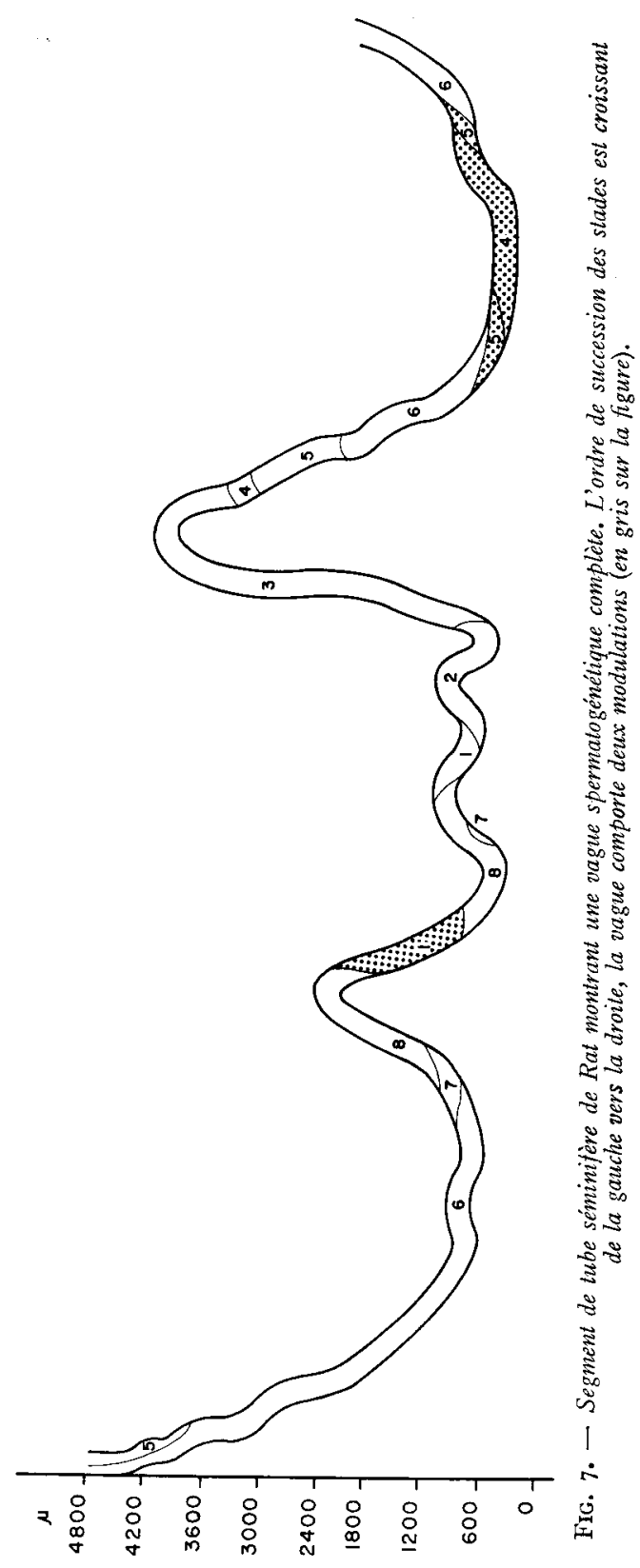


Les longueurs moyennes des segments occupés par les différents stades varient d'un stade à l'autre (tableaux I et 2). Ainsi chez le Rat, le stade 6 occupe une longueur moyenne de $3255 \mu$ alors que le stade I ne s'étend que sur $682 \mu$; chez le Taureau le stade I occupe une longueur moyenne de I $385 \mu$ et le stade 5 ne s'étend que sur $\mathrm{I} 45 \mu$.

\section{TABLEAU 2}

Comparaison des longueurs relatives du stade du cycle de l'épithélium séminifère et des fréquences relatives de ces stades déterminées directement sur coupes histologiques chez le Rat

\begin{tabular}{|c|c|c|c|c|c|c|c|}
\hline Stades & $\begin{array}{l}\text { plus petite } \\
\text { longueur }(\mu) \\
\text { de tube oc- } \\
\text { cupé par un } \\
\text { stade }\end{array}$ & $\begin{array}{l}\text { plus grande } \\
\text { longueur }(\mu) \\
\text { de tube oc- } \\
\text { cupé par un } \\
\text { stade }\end{array}$ & $\mid \begin{array}{c}\text { longueur } \\
\text { moyenne }(\mu) \\
\pm \text { erreur type }\end{array}$ & $\begin{array}{c}\text { nombre de } \\
\text { données }\end{array}$ & $\begin{array}{c}\text { Coefficient } \\
\text { de variation }\end{array}$ & $\begin{array}{l}\text { proportion } \\
\text { relative en } \\
\% \text { des lon- } \\
\text { gueurs } \\
\text { moyennes }\end{array}$ & $\begin{array}{c}\text { fréquence } \\
\text { relative en } \\
\% \text { (par la } \\
\text { méthode } \\
\text { directe) } \\
\pm \text { erreur type } \\
1724 \text { sections }\end{array}$ \\
\hline $1 \ldots \ldots$ & 247 & 1375 & $682 \pm 119$ & 11 & 58,3 & 5,37 & $2,46 \pm 0,45$ \\
\hline $2 \ldots \ldots$ & 210 & 1940 & $835+228$ & 9 & 81,9 & 6,57 & $5,88 \pm 0,37$ \\
\hline & 210 & 4460 & $2575 \pm 750$ & 6 & 71,1 & 20,27 & $17,40 \pm 0,92$ \\
\hline $4 \ldots$ & 540 & 1580 & $1032 \pm 136$ & 8 & 36,5 & 8,28 & $5,92 \pm 0,17$ \\
\hline $5 \ldots \ldots$ & 120 & 3220 & $1256 \pm 414$ & 8 & 93,0 & 9,89 & $9,56 \pm 0,56$ \\
\hline $6 \ldots \ldots$ & 890 & 9930 & $3255 \pm 949$ & 9 & 87,4 & 25,63 & $28,19 \pm 1,59$ \\
\hline $7 \ldots \ldots$ & 675 & 3195 & $1534 \pm 259$ & 10 & 53,4 & 12,08 & $10,88=0,26$ \\
\hline $8 \ldots \ldots$ & 400 & 3500 & $1509 \pm 262$ & 15 & 67,3 & 11,88 & $19,54 \pm 1,5$ \\
\hline
\end{tabular}

\section{$\left.3^{\circ}\right)$ LONGUEUR DE LA VAGUE SPERMatogénétíQUe}

L'étude de la longueur moyenne des segments de tubes occupés par chaque stade, nous permet de définir une longueur moyenne théorique de la vague spermatogénétique : elle est de $4340 \mu$ chez le Taureau et de r2 6 ro $\mu$ chez le Rat.

En fait cette longueur diffère de la longueur réelle de la vague par suite de l'existence de modulations. En pratique, nous avons trouvé des vagues plus longues que la vague théorique: 7 860 $\mu$ chez le Taureau, I4 9ro $\mu$ à 2I $382 \mu$ chez le Rat.

$4^{\circ}$ ) COMPARAison ENTRE IA LONGUEUR MOYENNE DES STADES

DU CYCLE DE L'ÉPITHÉLIUM SÉMINIFÈRE ET LEUR FRÉQUENCE RELATIVE

Entre divers animaux d'une même espèce, la constance des fréquences relatives des stades du cycle de 1'épithélium séminifère a été montrée par ORTAvANT (I958), ClERMONT et LEBLOND (I952). Ces fréquences relatives sont proportionnelles à la longueur des segments de tubes séminifères occupés par chacun des stades; nous venons de voir que les longueurs sont fortement variables. Cependant si nous comparons la fréquence relative déterminée directement avec celle obtentue à partir des longueurs moyennes des segments de tubes séminifères occupés par chacun des stades nous constatons que les deux séries de données coïncident assez bien dans 
chacune des espèces. Les différences trouvées au niveau du stade 8 chez le Rat sont dues vraisemblablement au nombre relativement peu élevé de données. Par conséquent, les fréquences relatives des stades ne sont que le reflet des longueurs moyennes occupées par les différents stades et pas du tout le reflet d'une constance quelconque de la longueur occupée par chacun des stades le long des tubes séminifères.

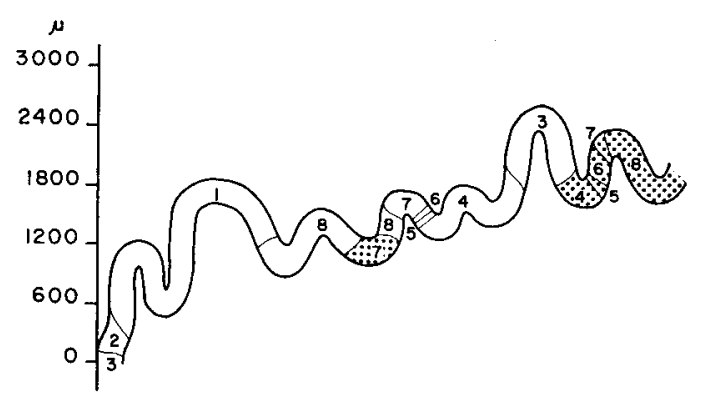

FIG. 8. - Segment de tube séminitère de Taureau montrant une vague spermatogénétique avec une modulation portant sur un seul stade. L'ordre de succession est décroissant de la gauche vers la droite

\section{DISCUSSION}

\section{Io) LA VAGUE SPERMATOGÉNÉTIQUE}

Nos résultats permettent d'affirmer que la vague spermatogénétique définie par REGAUD (IgOI) comme étant la succession dans un ordre numérique croissant ou décroissant de tous les stades du cycle de l'épithélium séminifère, existe aussi bien chez le Taureau que chez le Rat. Chez ces espèces, nous avons pu reconstituer des vagues spermatogénétiques entières. Si chez le Rat, nos résultats confirment ceux obtenus par PEREY, CLERMONT et LEBLOND (I96I) au moyen de techniques de reconstitution et avec une classification différentes de la nôtre, ils diffèrent de ceux de Kramer (1960) qui n'avait pu obtenir de vague spermatogénétique complète chez le Taureau, ayant reconstitué de trop courts segments de tube séminifère.

Il n'y a pas de modification du déroulement de la vague spermatogénétique même quand le tube séminifère présente un embranchement. La vague spermatogénétique est donc un phénomène général.

Cependant nous avons observé aussi bien chez le Taureau que chez le Rat la présence d'irrégularités mineures ou modulations qui perturbent la vague spermatogénétique sans en abolir le déroulement général. L'étude d'une certaine longueur de tube séminifère montre bien que même dans le cas de modulation portant sur plusieurs stades, il y a un gradient continu de cet ordre le long du tube comme nous l'avons observé. Nos résultats confirment ceux obtenus chez le Rat par PEREY, CLERMONT et LEBLOND (I96I) alors que KRAMER (I960) avait pensé que chez le Taureau il n'y avait pas un gradient continu de progression de la vague spermatogénétique mais que le tube se divisait en un certain nombre de petites unités possédant un " centre d'activité spermatogénétique ". 
Ces irrégularités déjà observées par CurTrs (I9I8) chez la Souris, le Lapin et le Chien ont été bien étudiées chez le Rat par Perey, Clermont et LEBLOND (I96I) qui ont introduit le terme de "modulation " et ont montré chez cet animal qu'elles ne détruisaient pas la continuité de la vague spermatogénétique. Ceci s'oppose aux résultats de CLELAND (I95I) chez le Cobaye qui pensait avoir observé l'existence de discontinuité dans l'ordre de succession des stades. Mais dans le cadre de son étude de la spermatogenèse cet auteur n'a pas défini de stades du cycle de l'épithélium séminifère et l'examen de ses propres reconstitutions de tubes séminifères permet de mettre en évidence des modulations plutôt que des discontinuités. Tibba (communication personnelle) chez le Taureau, à partir de coupes longitudinales de tubes séminifères conclut à la présence de discontinuité dans l'ordre de succession des stades. Cependant l'observation de ses coupes nous a permis de constater la présence de tous les stades intermédiaires entre deux stades donnés qui semblaient en discontinuité même lorsque ces stades occupaient une très petite surface de l'épithélium séminifère. Ceci peut se produire à tous les stades et représente une modu-

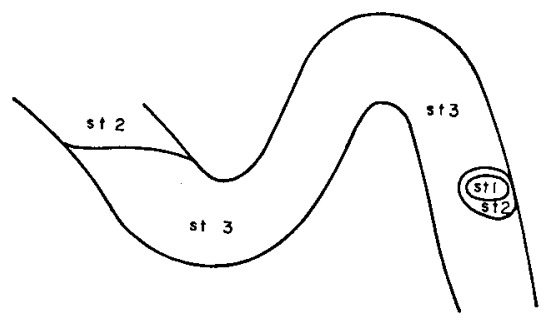

FIG. 9. - Segment de tube séminifère montrant une modulation poriant sur un nombre réduit de cellules

lation sur un nombre réduit de cellules de l'épithélium séminifère (fig. 9). Cependant au niveau de quelques cellules et non plus à celui des associations cellulaires il peut exister des discontinuités : par exemple, une spermatide au stade 4 peut parfois se trouver à côté d'une première division méïotique. Ceci a été trouvé aussi chez le Rat par Perei, Clermon'T et Leblond (I96I).

20) REIATION ENTRE IA LONGUEUR DES STADES DU CYCLE DE L'ÉPITHÉLIUM SÉMINIFÈRE ET LEUR FRÉQUENCE RELATIVE DÉTERMINÉE SUR COUPES HISTOLOGIQUES.

REGAUD (IgOI) pensait que la longueur de tube occupée par un stade était proportionnelle à la durée de ce stade dans le temps donc constante. Nos résultats ainsi que ceux de TibBa (communication personnelle) et de PEREY, CLERmont et LEBLOND (I96r) montrent au contraire que la longueur des différents segments de tube occupés par un même stade est variable. La longueur de la vague spermatogénétique est donc elle-même très variable ainsi que l'avait déjà observé VoN ĖBNER $(\mathrm{I} 87 \mathrm{I})$.

Cette variation de la longueur des segments de tube occupés par un stade n'est due ni à la présence de circonvolutions le long des tubes puisque nous avons effectué des corrections en fonction de celles-ci, ni aux techniques histologiques employées. Ceci conduirait à admettre que ni la durée d'un stade ni celle du cycle de l'épithé- 

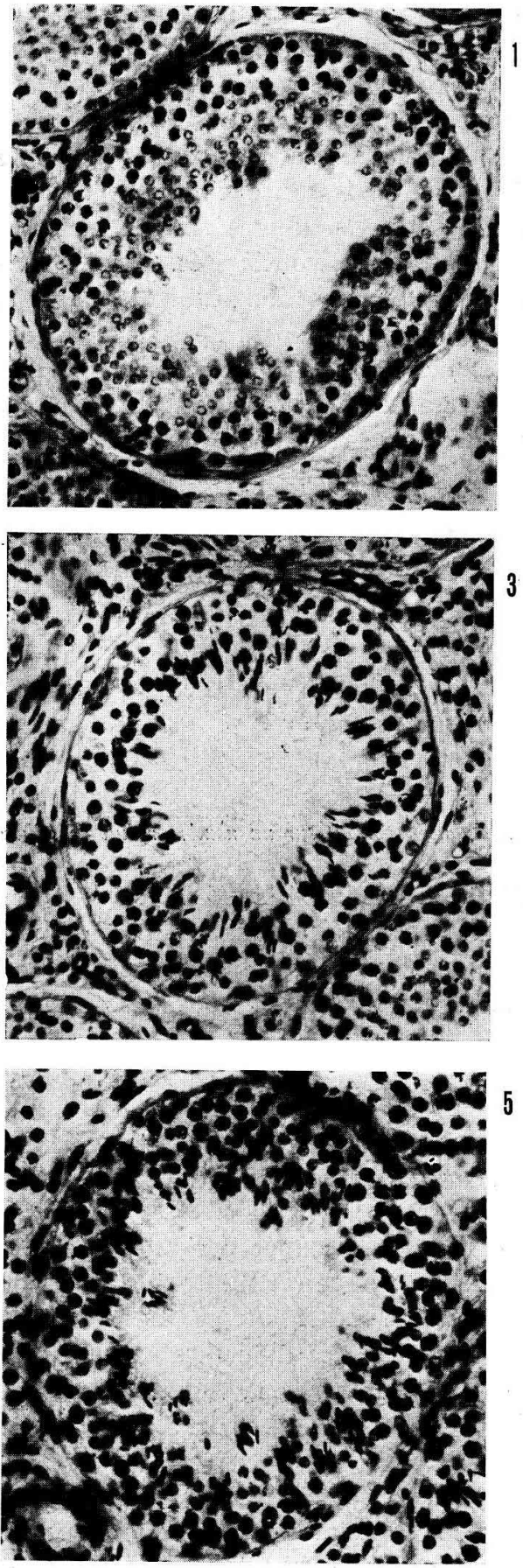
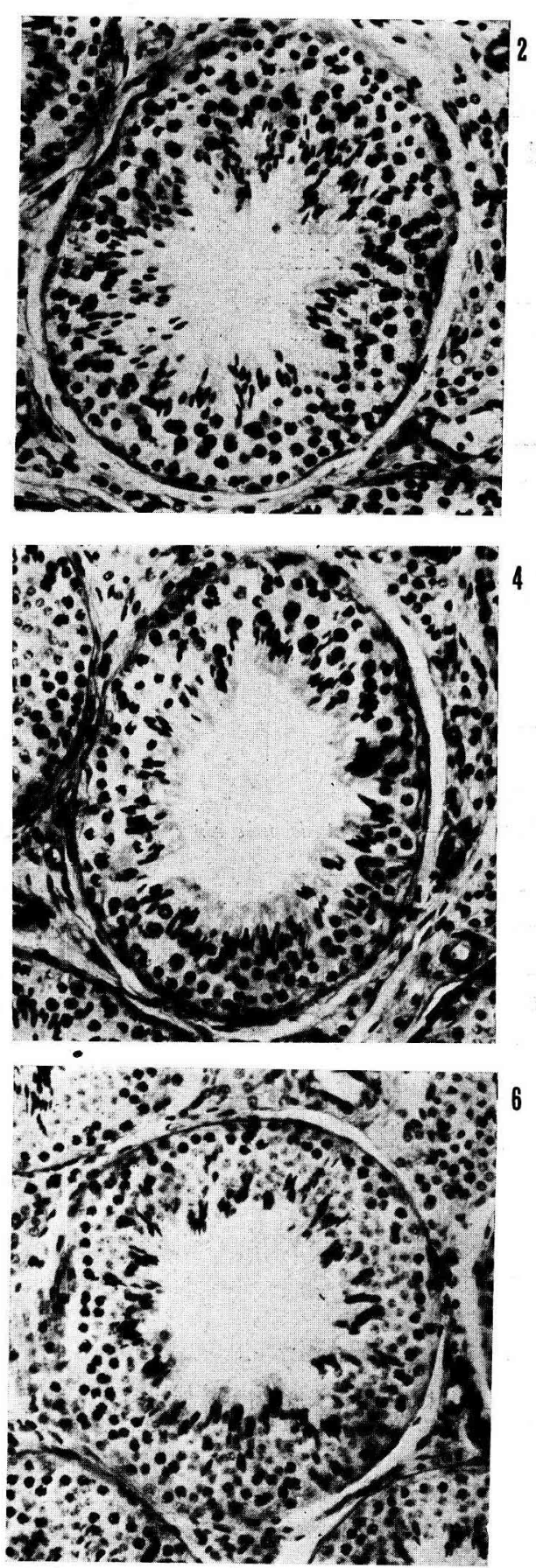

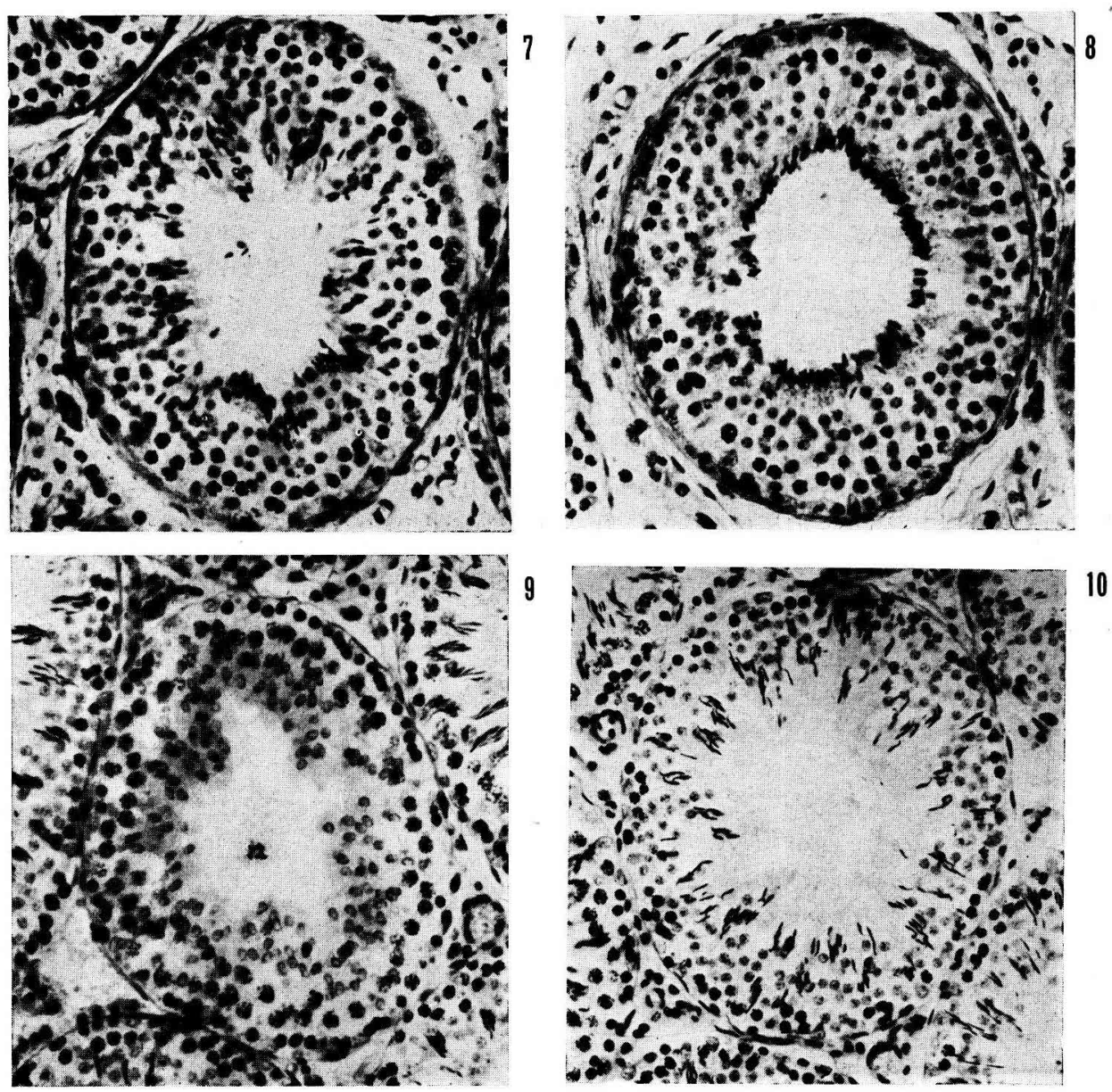

TAUREAU Stade I : Il commence dès la fin de la disparition des spermatozoïdes attachés à l'épithélium séminifère et se poursuit jusqu'au début de l'élongation et de l'augmentation de la colorabilité du noyau des spermatides. Ce stade est caractérisé par la présence de spermatides à noyaux ronds dont la chromatine ne se colore pas en une masse homogène $\left(n^{\circ} \mathrm{I}\right)$.

Siade 2 : Le noyaux de spermatides s'allongent et s'orientent vers la membrane basale des tubes séminifères $\left(\mathrm{n}^{\circ} 2\right)$.

Stade 3 : Les spermatides se groupent en faisceaux dont la pointe se dirige vers les noyaux des cellules de Sertoli $\left(\mathrm{n}^{\circ} \cdot 3\right)$.

Stade 4 : De la première division méiotique des spermatocytes I jusqu'à la demière division mitotique des spermatocytes II $\left(n^{\circ} 4\right)$.

Stade 5 : Le noyau des spermatides néoformées est petit et possède une chromatine croûtelleuse. Durant ce stade les faisceaux des spermatides allongées sont irrégulièrement répartis à l'intérieur de l'épithélium séminifère $\left(\mathrm{n}^{0} 5\right)$.

Stade 6 : La chromatine des noyaux des jeunes spermatides devient poussiéreuse : les faisceaux de spermatides allongées ont une répartition régulière dans l'épithélium séminifère et la majeure partie d'entre eux est encore attachée aux noyaux des cellules de Sertoli $\left(n^{0} 6\right)$.

Stade 7 : Les faisceaux de spernatides allongées émigrent vers la lumière. Ce stade se termine quand toutes les spermatides bordent la lumière $\left(n^{\circ} 7\right)$.

Stade 8 : Les spermatides, devenues spermatozoïdes, bordent la lumière du tube et sont progressivement éliminées $\left(n^{\circ} 8\right)$.

RAT Stade I : Le noyau des spermatides n'est plus exactement rond; cependant il n'y a pas de spermatides allongées et orientées vers la membrane basale $\left(\mathrm{n}^{\circ} 9\right)$.

Stade 5: La chromatine des jeunes spermatides ne présente pas les croûtelles caractéristiques du Taureau. Cependant le noyau de celles-ci est plus petit qu'au stade suivant. Les spermatides allongées ont une diposition irrégulière dans l'épithélium séminifère $\left(\mathrm{n}^{\circ} \mathrm{IO}\right)$.

Annales de Biologie animale. - I963. 
lium séminifère ne sont constants. Or, des travaux récents à l'aide de radio-isotopes ont montré que la durée des divers processus spermatogénétiques était constante pour une espèce donnée, ORTavan't (I958) chez le Bélier, Clermont, LEBLONd et Messier (I959) chez le Rat, OrGEBIN-Crist (I96r) chez le Taureau. Les stades, reflets des associations cellulaires, constituant le cycle de l'épithélium séminifère ont donc une durée constante; ainsi leur fréquence n'est que l'image des proportions relatives de la longueur moyenne de ces stades dans une vague spermatogénétique. Cette longueur moyenne doit, elle-même, être fixe pour un stade et une espèce donnés.

\section{$3^{\circ}$ ) Progression et origine de la VAGUe sPermatogénétique}

REGaUd (IgOI) émit l'hypothèse que la succession des stades dans l'espace ne se faisait pas selon une génératrice du tube mais selon une bande enroulée en hélice dont le pas était proportionnel à la durée relative des stades. Il avait basé cette théorie sur la présence pour une section circulaire de tube séminifère, d'une part de deux stades consécutifs et d'autre part de l'orientation hélicoïdale des flagelles des spermatozoïdes au stade 8. Or l'examen des segments de tubes séminifères ne nous a pas permis d'étayer cette hypothèse.

Si le mode de progression de la vague spermatogénétique n'est pas connu avec précision, $\mathrm{il}$ en est de même de son origine.

CLELAND (I95I) suppose que "la structure de la vague spermatogénétique typique ne pourrait être obtenue qu'après un fonctionnement continu et répété du tube ". Ceci tendrait à dire que la vague spermatogénétique n'existerait que chez des animaux âgés. Or nous avons étudié des taureaux pubères d'âge différent; chez les uns et les autres, nous avons trouvé l'existence de la vague spermatogénétique.

I a succession continue de plusieurs vagues spermatogénétiques le long du tube séminifère a été expliquée par PEREY, CLERMON' et LEBLOND (Ig6I) de la façon suivante : le tube séminifère serait divisé en un certain nombre d'unités dans lesquelles le démarrage a lieu concomitamment et dans lesquelles la vague spermatogénétique évolue dans un sens préférentiel ; les modulations existeraient dès le début de la spermatogenèse; quoi qu'il en soit cette origine ne pourra être étudiée que sur des animaux en cours de puberté.

\section{CONCLUSION}

Nous avons étudié la vague spermatogénétique sur 4 taureaux et 4 rats pubères par la technique de reconstitution des tubes à partir de coupes sériées. Le cycle de 1'épithélium séminifère formé par les différentes associations des cellules germinales est divisé en huit stades selon la classification de CuRTIS (I9I8), RoOSEN-RungE et Giesei, (1950) et Ortavant (I958).

Les résultats indiquent l'existence d'une continuité dans la succession numérique des stades du cycle de l'épithélium séminifère le long du tube : un segment de tube occupé par un stade déterminé est toujours précédé et suivi par des segments portant le numéro directement supérieur ou inférieur dans la classification définie 
plus haut. L'arrangement de ces segments aboutit à la formation de la vague spermatogénétique définie comme la série complète des huit stades du cycle de l'épithélium séminifère juxtaposés dans un ordre croissant ou décroissant.

La vague spermatogénétique peut cependant présenter des irrégularités appelées " modulations " qui sont des inversions locales de l'ordre de succession des stades, mais, même dans ce cas, les numéros des stades adjacents restent toujours immédiatement voisins.

Ces modulations peuvent porter sur un seul ou sur plusieurs stades.

Le long d'un même tube les vagues se succèdent selon un ordre préférentiel. La longueur du segment de tube occupé par un stade déterminé en plusieurs endroits d'un même tube ou sur des tubes différents est variable ; on peut cependant calculer la longueur moyenne de chaque stade.

Par suite des variations de la longueur de segment de tube occupé par chaque stade et de l'existence de modulations il en résulte une certaine variabilité de la longueur de la vague spermatogénétique. Mais on peut l'évaluer très approximativement à Io $\mathrm{mm}$ chez le Taureau et à $20 \mathrm{~mm}$ chez le Rat.

Ces résultats ont été discutés en fonction de la fréquence relative des stades calculée directement sur des coupes histologiques. Nous avons trouvé que la longueur moyenne relative des segments de tubes séminifères occupés par les différents stades correspond assez bien avec la fréquence relative de ces stades dans les deux espèces. Mais il n'y a pas de relation entre les longueurs individuelles des différents segments de tubes séminifères occupés par un stade déterminé et la durée de ce stade qui est constante. La vague spermatogénétique n'est donc pas dans l'espace ce que le cycle de l'épithélium séminifère est dans le temps.

Reçu pour publication en février 1963 .

SUMMARY

A COMPaRative STUDY OF TIIE SPERMatogenETIC WAVE IN THE BULL AND THE RAT

The arrangement of the stages of the cycle of the seminiferous epithelium in adult and fertile bulls and rats had been studied. The technique employed was that of reconstituting the seminiferous tubules after histological sectioning in series, after which the nature of the stages and the lenght of the tube affected by these were determined.

Results showed that there was a continuity in the numerical succession of the stages and the existence of the complete spermatogenetic wave as defined by REGAvI. However, this wave could show modulations which were due to local inversions of the order of succession of the stages, without the numbers of the stages being for that matter immediate neighbours. Once the modulation was accomplished the wave returned to its normal course. The degree of this modulation was variable : it could affect a varying number of stages, but this number was always odd.

The individual lengths of the different segments occupied by a given stage were not regular within the testicule, for instance, stage 4 in the bull varied from $60 \mu$ to $1720 \mu$. Furthermore, the average length of the segments occupied by the different stages differed from one stage to another.

As the result of this variation in the length of the segments of the tube occupied by each of the stages and of the modulations, there occurred a certain variation in the length of the spermatogenic wave which could be measured very approximately at $10 \mathrm{~mm}$ in the bull and $20 \mathrm{~mm}$ in the rat.

The average relative lengths of the segments of the seminiferous tubules occupied by the dif. ferent stages corresponded approximately with the relative frequencies of these stages in the two species, but there was no relationship between the individual lengths of the different segments of the seminiferous tubule occupied by any given stage and the length of that stage, which latter was a constant. 


\section{RÉFÉRENCES BIBLIOGRAPHIQUES}

Amman R. P., I96r. Reproductive physiology of the male bovine. Ph. D. thesis Pennsylvania State University, University Park. 206. p.

BENDA C. C., I887. Untersuchungen über den Bau des Functionierenden Samenkänelchens einiger säugetiere und Folgerungen für spermatogenese dieser Werbelthlerklasse. Arch. Mikr. Anat., 30, 49-1 1o.

Cleland K. W., 1951. The spermatogenetic cycle of the Guinea Pig. Aust. J. Sci. Res., 34, 344-369.

Clermont Y., Le3lond C. P., I952. Définitions of the stages of the cycle of the seminiferous epithelium in the Rat. Ann. N. Y. Acad. Sci., 55-64, 548-573.

Clermont Y., Hrckins C., ig6r. Microscopic anatomy of the sex cords and seminifurous tubules in growing and adult male albino rats. Am. J. Anat., 108, 79-98.

Clermont Y., leblond C. P., Messier B., i959. Durée du cycle de l'épithélium séminal du Rat. Arch. Anat. Micr. Morphol. Exper., 48 bis, 37-56.

Curtis G. M., 1918. The morphology of the Mammalian seminiferous tubule. Amer. J. Anat., 24, $339-394$.

EBNer H. Von, I87. Untersuchung über den Bau der Samenkähalchen und die entwick der spermatozoïden bei den säugetiesen und bein Henschen. Rollet's Unter Inst. f. Physiol. Histol. in graz., p. 200.

Ebner H. Von, 1888. Zur spermatogeneses bei den siengetiesen. Arch. Mikr. Anat., 31, 236-292.

Fürst C., I887. \& Über die Entwicklung der Samen körpeichen bei den Beutelthieren. Arch. Mikr. Anat., 30, 33 .

Kramer M. F., 1960. Spermatogenesis bij de stier. Dr. Diergeneesk. Ryksuniversiteit te. Utrecht. I83 p.

Mowry R. W., I96o. Revized method producing improved coloration of acidic polysaccharid with Alcian blue $8 \mathrm{GX}$ supplied currently. Proc. J. II istochem. Cytochem., 8, 323.

ORGEBIN-CRIST M. C., I96I. Recherches expérimentales sur la durée de passage des spermatozoïdes dans l'épididyme du Taureau. Thèse Doct. Univ. Lyon, 66 p., parue dans Ann. Biol. Bioch. Biophys., 1962, 2, 51-108.

Ortavant R., 1958. Le cycle spermatogénétique chez le Bélier. Thèse Doct., Paris, in7 p.

Perey B., Clermont Y., Leblond C. P., 1961. The waves of the seminiferous epithelium in the rat. Amer. J. Anat., 108, 47-78.

REGAUD B., igor. Études sur la structure des tubes séminifères et sur la spermatogenèse chez les Mammifères. Arch. Anal. Micr., 4, 10 I-1 56, $231-380$.

Roosen-Runge E. C., Giesel L. O., 1950. Quantitative studies on spermatogenesis in the albino rat. Amer. J. Anat., 87, I-zo.

TiвbA. Communication personnelle. 